\title{
Percepción sobre la Eutanasia en Estudiantes de Medicina de dos Universidades del Paraguay, $2017^{4}$ Perception on Euthanasia in Medical Students of two Uni- versities of Paraguay, 2017
}

\author{
Roberto Daniel Álvarez Ovelar; Marcos David Martínez Román; \\ Karen Yohana Rodas Benítez; Juan Manuel Invernizzi Prats ${ }^{5}$.
}

\section{DOI: https://doi.org/10.36003/Rev.investig.cient.tecnol.V3N1(2019)2}

\section{RESUMEN}

La eutanasia es el acto de acabar con la vida de un paciente a petición expresa de él, por padecer un sufrimiento sin perspectiva de cura, esta práctica es muy discutida y confronta principios de la bioética. En la mayoría de los países no es permitida pero su estudio y aprobación se está extendiendo. Los objetivos del trabajo fueron determinar la percepción de la eutanasia en estudiantes de medicina de la Universidad Nacional de Caaguazú (UNCA) y la Universidad Católica de Asunción (UCA) durante el año 2017. Se realizó un estudio observacional descriptivo de corte transversal con muestreo probabilístico por conglomerados, el instrumento fue un cuestionario validado por expertos. Se utilizaron las medidas de tendencia central, dispersión y proporciones. Fueron incluidos 317 estudiantes de medicina, el 66\% fueron de sexo femenino, la edad de los estu-

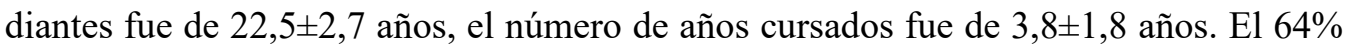
de todos los estudiantes se mostró a favor de la eutanasia (61\% de la UNCA y $66 \%$ de la UCA), el 48\% considera a la eutanasia como una forma de muerte digna. El 57\% de los varones estarían dispuestos a practicar la eutanasia. E1 94\% de los agnósticos se encontró a favor de la eutanasia. Más de la mitad de los estudiantes de medicina se encontraron a favor de la eutanasia y la mitad la consideró como una muerte digna. Los varones y los agnósticos representaron una mayor proporción de estudiantes a favor de la eutanasia.

Palabras clave: Eutanasia/ética; Estudiantes de Medicina; Discusiones Bioéticas

${ }^{4}$ Fecha de recepción: abril 2019; fecha de aceptación: mayo 2019

${ }^{5}$ Médicos Residentes de Medicina Interna del Hospital Central del Instituto de Previsión Social. Asunción, Paraguay. 


\section{ABSTRACT}

Euthanasia is the act of ending the life of a patient at the express request of him, for suffering a disease without perspective of cure, this practice is very discussed, confronts principles of bioethics. In most countries, it is not allowed but its study and approval is spreading. The objective wasto determine the perception of euthanasia in medical students of the National University of Caaguazú (UNCA) and the Catholic University of Asunción (UCA) during the year 2017. A cross-sectional descriptive observational study was carried out with probabilistic sampling by conglomerates, the instrument was a questionnaire validated by experts. Measures of central tendency, dispersion and proportions were used. Three hundred and seventeen medical students were included, $66 \%$ were female, and the students' age was $22.5 \pm 2.7$ years, the number of years studied was $3.8 \pm 1.8$ years. $64 \%$ of all students were in favor of euthanasia $(61 \%$ from UNCA and $66 \%$ from UCA), $48 \%$ of all students considered euthanasia as a dignified dead. $57 \%$ of men would be willing to practice euthanasia. $94 \%$ of agnostics found themselves in favor of euthanasia. More than half of medical students found themselves in favor of euthanasia and considered euthanasia as a dignified dead. Males and agnostics represented a higher proportion of students in favor of euthanasia.

Keywords: Euthanasia / ethics; Medicine students; Bioethical Discussions. 


\section{INTRODUCCIÓN}

La palabra eutanasia etimológicamente proviene del griego "euthanatos" que significa "buen morir" (1). Consiste en el acto de acabar con la vida del paciente a petición expresa de él para terminar el sufrimiento propio de alguna enfermedad sin perspectiva de cura. Una definición más completa y actualizada es la siguiente: "en el contexto clínico, consiste en todas aquellas acciones realizadas deliberadamente por un médico, u otro profesional sanitario bajo su dirección, que tienen como objetivo provocar directamente la muerte a petición expresa, voluntaria y reiterada de un paciente capaz que presenta un sufrimiento intenso, físico o psíquico, a consecuencia de una enfermedad terminal o irreversible y que el propio paciente experimenta como algo inaceptable, insoportable e indigno"

(2).Estas acciones se realizan de acuerdo con la voluntad del paciente, esto último es lo que diferencia la eutanasia del homicidio. Esta práctica se registra desde los inicios de la medicina y ha generado discusiones presentes hasta nuestros días. Condenada por muchas religiones y por el juramento Hipocrático que manifiesta: «nunca daré un veneno a un paciente aunque me lo pidiere» (3). Esto promovió el rechazo de la mayoría de los médicos, tanto así que la Asociación Médica Mundial (AMM) se pronunció rechazando todo tipo de forma de eutanasia e instando a los médicos de países que respeten el curso natural de vida de los pacientes, aun disponiendo del marco legal para practicar la eutanasia (4).

Entre los argumentos de las personas que apoyan la eutanasia se destaca que la decisión de dónde y cuándo morir es una de las más privadas y personales de cada persona y ¿cómo podemos denegar la solicitud de un paciente de morir antes que después? Entonces, si respetar la decisión de los pa- cientes fuera la premisa, ¿por qué negar la solicitud de eutanasia a una persona que no padece una enfermedad terminal pero desea morir por otras razones, como alguna dolencia tolerable y tratable o la pérdida de un ser querido? Fundamentarse en la autonomía para la eutanasia genera una "pendiente resbaladiza" con consecuencias tétricas $(5,6)$.

La discusión sobre la eutanasia, su legislación y su moralidad, es un tópico constante presente en todos los países y en todos los tiempos (7). En la Grecia antigua esta palabra significaba una muerte suave, honorable, sin dolor, como la describe Homero en su solemne obra La Odisea. Durante el régimen nazi, Adolf Hitler firmó en 1939 un "decreto de eutanasia" en el que autorizaba a médicos a seleccionar con criterio a "enfermos incurables" incluyendo niños con defectos hereditarios y adultos improductivos, a fin de otorgarles "una muerte misericordiosa" (8) aunque la finalidad era económica y a favor de la eugenesia, se reportaron 275.000 casos de eutanasia no voluntaria, incluyendo la extensión del programa a los campos de concentración (9).

La relevancia del presente trabajo radica en que más del $60 \%$ de las causas de muerte en Paraguay la constituyen las enfermedades cardiovasculares, cáncer y diabetes (10) enfermedades crónicas no transmisibles que engloban al $80 \%$ de todos los pacientes terminales (8) que solicitan la eutanasia en otros países. Si además consideramos a los accidentes de tránsito, el otro gigante de las problemáticas de salud pública, vemos que es causa importante de discapacidad (11), entre los más importantes motivos de petición de eutanasia se encuentra el sentimiento de inutilidad. Cabe recalcar además que la esperanza de vida en Paraguay presenta un aumento progresivo anual (10) lo que genera un aumento de la población de la tercera edad con sus 
problemas de salud consecuentes.

La eutanasia es una práctica legalizada en países como Holanda, Bélgica, Luxemburgo, Canadá y Colombia; en Suiza y ciertos estados de Estados Unidos existen legislaciones que avalan la eutanasia y el suicidio asistido(12), asimismo su implementación en varios países está siendo estudiada y es motivo de debate (13).

Debido a la laxitud de la legislación en Suiza, el turismo suicida en este país ha doblado la frecuencia de casos en los últimos años(14), considerando que la mayoría de personas que solicitan el suicidio asistido en Suiza provienen de otros países de Europa (15). La facilidad que presenta Suiza para esta práctica es un hecho que preocupa a la Corte Europea de los Derechos Humanos ya que un importante porcentaje de casos en Dignitas (centro especializado en muerte asistida, en Suiza) no es a causa de una enfermedad terminal, sino por "cansancio de la vida" (14). Como no existe un consenso siquiera sobre la definición, ciertos autores definen la eutanasia como "la muerte producida intencionalmente del modo menos doloroso posible, motivado exclusivamente por la estimación de que así se logra el mejor interés de la persona que muere." (16). Esta última definición requiere un análisis especial ya que han suprimido como requisito indispensable contar con la voluntad del paciente, sino que se produce la muerte suponiendo que es lo mejor para el enfermo, catalogándola como eutanasia no voluntaria.

La percepción de la eutanasia es muy dispareja comparando entre países, médicos y estudiantes de medicina $(17,18$, 19,20).

En Estados Unidos el porcentaje de apoyo a la eutanasia en la población general se ha mantenido en un rango de $47-69 \%$, en Europa occidental se ha descrito un importante aumento del apoyo, mientras que en Europa central y oriental ha disminuido.
En Paraguay no existen publicaciones que describan la percepción sobre la eutanasia en la población en general ni estudiantes de medicina o médicos.

Existen varios factores que influyen en la percepción de la eutanasia que varían de un país a otro. Como la religión, el marco legal, la valoración de la muerte. El agnosticismo y el género masculino son factores asociados a una percepción positiva de la eutanasia (16).Es difícil estudiar la percepción y el comportamiento de personas ante una práctica que se encuentra prohibida en el país y más aún si se trata de un tema tan delicado como el cuidado de la vida de una persona.

El aumento de la cantidad de muertes producidas por enfermedades crónicas produce una mayor consideración a los cuidados en el final de la vida, desarrollándose así toda una especialidad, la Medicina Paliativa, sin embargo a la vez se plantea ofrecer al enfermo terminal la posibilidad de decidir el momento de su muerte, cuya práctica implicaría una infracción de los médicos al juramento hipocrático que reza «nunca daré un veneno a un paciente aunque me lo pidiere»

En Paraguay la eutanasia no es permitida, mientras la cantidad de casos van aumentando e introduciéndose en varios países $(12,14)$. Por todo lo anterior, surge como pregunta: ¿Cuál es la percepción sobre la eutanasia de los estudiantes de medicina de la Universidad Nacional de Caaguazú (UNCA) y la Universidad Católica Nuestra Señora de la Asunción (UCA) durante el año 2017?

\section{MÉTODO}

Se realizó un estudio de tipo observacional, descriptivo, de corte transversal. El universo de estudio estuvo conformado por estudiantes de Medicina de la Universidad Nacional de Caaguazú (UNCA) y la Universidad Católica Nuestra Señora de Asun- 
ción (UCA) sede Asunción. La muestra fue recogida por muestreo probabilístico por conglomerados bietápico, en estudiantes de medicina matriculados al año lectivo 2017. El total del tamaño de la muestra para cada universidad fue dividido en seis, lo que corresponde a cada curso. Mediante una planilla con la lista de alumnos, se utilizó el paquete epidemiológico EPIDAT $4.2{ }^{\circledR}$ para seleccionar de manera aleatoria los participantes para cada subgrupo hasta completar el tamaño muestral requerido por universidad. Para el cálculo del tamaño de la muestra de estudiantes de la UNCA se utilizó el paquete epidemiológico EPIDAT $4.2 \AA$, considerando un tamaño poblacional de 246; un nivel de confianza de $95 \%$, una proporción esperada $50 \%$ y una precisión del 7\% arrojó un tamaño muestral recomendado de 110 estudiantes. Se incluyeron 119 sujetos.Considerando un tamaño poblacional de 815 estudiantes de la UCA, un nivel de confianza de $95 \%$, una proporción esperada $50 \%$ y una precisión del 7\% arrojó un tamaño muestral recomendado de 159 estudiantes. Se incluyeron a 198 sujetos. Como criterios de inclusión: Estudiantes de medicina del primero al sexto curso matriculados al año lectivo 2017 de las Universidad Católica Nuestra Señora de Asunción y la Universidad Nacional de Caaguazú que hayan accedido a participar del estudio firmando un consentimiento informado.

Para la recolección de los datos se empleó un cuestionario adaptado de García Hirsch et al (alfa de Cronbach 0,84), validado por expertos para la ocasión. Este instrumento estuvo dividido en 2 secciones: - la primera corresponde a variables socio demográficas y académicas, incluyendo preguntas cerradas y abiertas. - la segunda sección evalúa la percepción sobre la eutanasia con dos preguntas cerradas y 7 preguntas con escala tipo Likert.Todos los datos proveídos por las encuestas fueron tratados con confidencialidad, igualdad, y justicia. No existen riesgos para los sujetos incluidos en el estudio. No se realizó ningún tipo de discriminación étnica, económica ni social en base a los datos registrados en los cuestionarios, aspecto muy importante considerando que la eutanasia es prohibida en nuestro país y eso podría condicionar las respuestas de los estudiantes. La participación fue libre y voluntaria, previo consentimiento informado firmado por los alumnos. No se declaran conflictos de intereses. 


\section{RESULTADOS}

Participaron 317 estudiantes de medicina. De la totalidad de los estudiantes participantes, el 62\% (198) perteneció a la Universidad Católica de Asunción. E1 66\% (209) de los participantes del estudio era del sexo femenino con una distribución similar entre las dos muestras, en la UCA el $69 \%$ de los participantes fue de género femenino y en la Universidad Nacional de Caaguazú dicho género corresponde al $60 \%$. Con relación al estado civil, 295 encuestados (93\%) eran solteros, 12 se encontraban en unión estable $(3,8 \%)$, 9 casados (3\%) y uno divorciado $(0,3 \%)$. (Tabla 1$)$

TABLA 1. Distribución de los estudiantes de Medicina de la Universidad Nacional de Caaguazú y la Universidad Católica "Nuestra Señora de la Asunción” de acuerdo a la actitud ante la eutanasia, 2017. $n=301$

\begin{tabular}{lrrr}
\hline Datos demográficos & UNCA & UCA & TOTAL \\
\hline Participantes & $\mathbf{3 8 \%}(\mathbf{1 1 9})$ & $\mathbf{6 2 \%}(\mathbf{1 9 8 )}$ & $\mathbf{3 1 7}$ \\
\hline Sexo & & & \\
Masculino & $40 \%(48)$ & $31 \%(60)$ & $108(34 \%)$ \\
Femenino & $60 \%(71)$ & $69 \%(138)$ & $209(66 \%)$ \\
Total & $100 \%(119)$ & $100 \%(198)$ & $317(100 \%)$ \\
Estado civil & & & \\
Soltero/a & $92,2 \%(110)$ & $93 \%(185)$ & $295(93 \%)$ \\
Casado/a & $3,5 \%(4)$ & $3 \%(5)$ & $9(3 \%)$ \\
Unión estable & $3,5 \%(4)$ & $4 \%(8)$ & $12(3,7 \%)$ \\
Divorciado/a & $0,8 \%(1)$ & $0 \%$ & $1(0,3 \%)$ \\
Total & $100 \%(119)$ & $100 \%(198)$ & $317(100 \%)$
\end{tabular}

Fuente: Encuesta sobre percepción de eutanasia en estudiantes de medicina de la Universidad Nacional de Caaguazú y la Universidad Católica "Nuestra Señora de la Asunción", 2017.

Del total de estudiantes participante en este estudio el 67,2\% (213) tenía una edad comprendida entre 20 a 24 años de edad, el 17,6\% (56) entre 25 a 29 años, y $13,5 \%$ (42) entre 18 a 19 años de edad.

Considerando toda la muestra, el $64 \%$ de los estudiantes se mostraron a favor de la eutanasia, (El $61 \%$ de los estudiantes de la UNCA estuvieron a favor de la eutanasia, así como el $65 \%$ de los estudiantes de la UCA). (Tabla 2). Considerando la distribución por géneros, El $60 \%$ de las mujeres se mostró a favor de la eutanasia, mientras que en los hombres el porcentaje a favor fue mayor, $71 \%$. Considerando las edades de los estudiantes de medicina, El 72\% de los estudiantes de 18 y 19 años se encontró a favor de la eutanasia, en el grupo etario de mayor frecuencia (20 - 24 años) la actitud a favor de la eutanasia fue del $61 \%$ de los estudiantes. 
TABLA 2. Distribución de los estudiantes de Medicina de la Universidad Nacional de Caaguazú y la Universidad Católica "Nuestra Señora de la Asunción” de acuerdo a la actitud ante la eutanasia, 2017. $n=301$

\begin{tabular}{lcrr}
\hline Eutanasia & UNCA & UCA & TOTAL \\
\hline A favor & $61 \%(48)$ & $65 \%(120)$ & $64 \%(193)$ \\
En contra & $39 \%(46)$ & $35 \%(62)$ & $36 \%(108)$ \\
\hline Total & $100 \%(119)$ & $100 \%(198)$ & $100 \%(301)$ \\
\hline
\end{tabular}

Fuente: Encuesta sobre percepción de eutanasia en estudiantes de medicina de la Universidad Nacional de Caaguazú y la Universidad Católica "Nuestra Señora de la Asunción”, 2017.

El 52\% de los participantes no consideró que la eutanasia sea un homicidio. E1 21\% consideró que sí lo es. Mientras que 80 estudiantes $(27 \%)$ se mostraron neutrales. El $29 \%$ de los encuestados valoraron la eutanasia como pecado, mientras que el $45 \%$ no lo consideró como tal. 146 estudiantes (48\%) de medicina encuestados consideraron a la eutanasia como una muerte digna. El 30\% no estuvo de acuerdo con que sea una muerte digna.El 55\% de los estudiantes (166) no consideró que la eutanasia ocasionaría una devaluación de la profesión médica. 75 estudiantes (25\%) estuvieron de acuerdo con que sí se produciría una devaluación de la profesión.El 46\% de los encuestados se mostraron de acuerdo con que es preferible morir antes que vivir con una calidad de vida inadecuada, el
$30 \%$ se mostró en contra. El 50\% de los estudiantes manifestaron que sí practicarían la eutanasia. Del total de los participantes el 93\% eran solteros, de los cuales el 63\% se manifestó a favor de la eutanasia así como el $92 \%$ de los que se encontraban en unión estable, que representaron el 3,7\% del total de la muestra.

El $68 \%$ de los estudiantes del tercer y quinto año se mostraron a favor de la eutanasia así como el 55\% de los médicos internos (sexto año de la carrera).Con relación a las creencias religiosas, el 94\% de los agnósticos se mostró a favor de la eutanasia, así como el 64\% de los católicos. El 56\% de los evangélicos se manifestó en contra de la eutanasia. La religión de mayor frecuencia fue la católica, representando al $78 \%$ de todos los participantes.

\section{DISCUSIÓN}

En el presente trabajo, el $64 \%$ de estudiantes de medicina participantes se encuentra a favor de la eutanasia. Los estudiantes de la UNCA están a favor en un $61 \%$ y los de la UCA en un $66 \%$. Estos resultados muestran similitud con estudios realizados en Perú (16) y Venezuela, pero contrastan con otros realizados en México (20) y Alemania (17), donde el porcentaje a favor de la eutanasia es menor. En la población general, estos últimos años se ha producido un aumento en el apoyo a la eutanasia. La percepción positiva de la eutanasia varía entre 47- 69\% en Estados Unidos y Europa. Vivir una experiencia tal, como ver a un ser querido en estado terminal sufriendo dolores insoportables y morir de manera angustiosa podría instar a una percepción positiva de la eutanasia como alternativa al sufrimiento.Con relación al género, el 71\% de los hombres se mostró a favor de la eutanasia y el 57\% estaría dispuesto a practicarla como médicos, el $60 \%$ de las mujeres se mostraron a favor de la eutanasia y solo el $46,5 \%$ de ellas lo practicaría. Estudios previos han señala- 
do que los hombres están más dispuestos a practicar la eutanasia, lo cual concuerda con el presente estudio. Otro resultado interesante es la diferencia encontrada entre la percepción y la posible práctica de la eutanasia, el $64 \%$ se encuentra a favor pero solo el $50 \%$ lo practicaría. Resulta muy difícil valorar una acción como ésta, considerando que esta práctica no es legal en Paraguay, lo cual podría ser determinante al momento de decidir una postura a favor o en contra de la eutanasia.Con respecto a las creencias religiosas, el $78 \%$ de los participantes son católicos, de los cuales el $64 \%$ se encuentran a favor de la eutanasia. Lasegunda en frecuencia es la evangélica, que representa el $11 \%$ de los estudiantes y de éstos, el $56 \%$ están en contra de la eutanasia. El 13\% de los que están a favor de la eutanasia son ateos y agnósticos. El 82\% de los ateos se encuentran a favor de la eutanasia así como el $94 \%$ de los agnósticos. El 29\% de toda la muestra considera la eutanasia como pecado. En México se encontró que el catolicismo es el mayor factor religioso asociado a una percepción negativa de la eutanasia, mientras que en Perú el agnosticismo es un factor asociado a una percepción positiva de la eutanasia (1).

Estudios previos han señalado que a medida que aumenta los años estudiando medicina, aumenta la percepción positiva de la eutanasia (1). En el presente trabajo el $53 \%$ de los estudiantes del primer año se encuentra a favor de la eutanasia, así como el $58 \%$ del segundo año, $68 \%$ del tercer año, $64 \%$ del cuarto año, $68 \%$ del quinto año y en los médicos internos el porcentaje a favor es de $55 \%$. Vale mencionar dos puntos: el primero, por las características del estudio de corte transversal es imposible realizar un seguimiento a la percepción de cada estudiante conforme vaya avanzando en la carrera y las prácticas hospitalarias se intensifican en los últimos años hasta llegar al internado rotatorio, donde se desarrolla un año entero en el hospital, tratando con los pacientes y sus familiares, antesala de lo que será el día a día de mañana. El segundo punto a considerar, se observa un decremento de $13 \%$ entre la cantidad de estudiantes a favor de la eutanasia del quinto y sexto curso (Internado rotatorio).

El $52 \%$ de los estudiantes no considera que la eutanasia sea un homicidio. El Código penal de Paraguay cataloga como homicidio por compasión, penado con 6 meses hasta 3 años de prisión.La medicina desde sus inicios ha sido considerada como la noble profesión del cuidado de la vida de las personas. El arte de curar o en su defecto consolar y brindar compañía. E1 55\% de los estudiantes de medicina no considera que la práctica de la eutanasia ocasionaría una devaluación de la profesión médica. La cuestión sería qué responde la población general ante esta pregunta.

Si bien el $64 \%$ de los estudiantes están a favor de la eutanasia, solo el $50 \%$ de todos practicaría la eutanasia como médicos. Esta diferencia podría deberse a varios factores. Como mencionamos, es difícil evaluar una práctica que es prohibida en el entorno. La pregunta no condicionaba la práctica de la eutanasia en un marco legal, por lo tanto la cantidad de futuros médicos que la practicaría podría ser mayor si estuviera permitida. 


\section{CONCLUSIONES}

Predominaron estudiantes de género femenino, el estado civil soltero/a, la religión católica y estudiantes con edades comprendidas entre 20 - 24 años. La mayoría de los estudiantes de medicina de la Universidad Nacional de Caaguazú (UNCA) y la Universidad Católica de Asunción (UCA) estuvieron a favor de la eutanasia. La percepción positiva fue mayor en los estudiantes de la UCA. Casi la mitad de los estudiantes consideró la eutanasia como una forma de muerte digna, la mayoría no la consideró como pecado u homicidio, tampoco pen- saron que la eutanasia ocasionaría una desvalorización de la profesión médica. La mitad de los estudiantes de medicina practicarían la eutanasia como médicos. Los hombres presentaron una mayor percepción positiva a favor de la eutanasia, así como los estudiantes de 18 y 19 años y de 25 a 29 años. Casi la totalidad de los agnósticos se mostraron a favor de la eutanasia, más de la mitad de los católicos estuvo a favor de la eutanasia mientras que la mayoría de los evangélicos se mostraron en contra de esta práctica.

\section{BIBLIOGRAFÍA}

1. García Hirsch SMI, Guevara Peralta RJ. Percepción de la Eutanasia y factores asociados, en estudiantes de Medicina de la Facultad de Ciencias de la Salud de la Universidad Peruana de Ciencias. 2014 [citado 23 de julio de 2017]; Recuperado a partir de: http://repositorioacademico.upc.edu.pe/upc/handle/10757/322312

2. Beca JPI, Leiva AL. ¿Podría ser aceptable la eutanasia infantil? RevChilPediatr [Internet]. 2014; 85(5):608-12. Disponible en: http://www. scielo.cl/scielo.php?script $=$ sci_arttext\&pi$\mathrm{d}=\mathrm{S} 0370-41062014000500013 \& \operatorname{lng}=\mathrm{es}$

3. Carrasco M Víctor Hugo, Crispi Francisca. Eutanasia en Chile: una discusión pendiente. Rev. méd. Chile [Internet]. 2016 Dic [citado 2017 Jul 31]; 144(12): 1598-1604. Disponible en: http://www. scielo.cl/scielo.php?script $=$ sci_arttext\&pid=S0034 98872016001200012\&ln$\mathrm{g}=$ es. $\quad$ http://dx.doi.org/10.4067/S003498872016001200012.

4. Asociación Médica Mundial: Resolución sobre la eutanasia. Bali: AMM; 2013. Disponible en: https:// www.wma.net/es/policies-post/resolucion-de-la-amm-sobre-la-eutanasia/

5. Saunders W, Fragoso M. Should We Legalize Voluntary Euthanasia and Physician Assisted Suicide? [Internet]. FamilyResearch Council. 2013 [citado 29 de octubre de 2017]. p. 1-6. Disponible en: http://downloads.frc.org/EF/EF09F59.pdf

6. Rodríguez Sui Qui MP. La actitud de los médicos ante la eutanasia y el suicidio medicamente asistido en la Ciudad de México [Internet]. Universidad Anáhuac; 2017. Disponible en: http://repositoriotesis.anahuac.mx/handle/123456789/931

7. Tannehill W. Sketches of the History of Literature: From the Earliest Period to the Revival of Letters in the Fifteenth Century. reimpresa. BiblioLife, editor. 2016. 98-100 p.

8. Friedlander $H$. The Origins of Nazi Genocide: From Euthanasia to the Final Solution. H-Holocaust [Internet]. 2000; Disponible en: https://web.archive. org/web/20090329020722/http://www. ess.uwe.ac.uk/genocide/reviewsh35.html 
9. Strzelecka I. Medical Crimes. The experiments in Auschwitz. 2.a ed. Oświęcim: International Center for education about Auschwitz and the Holocaust; 2016. 29-33 p.

10. Ministerio de Salud Pública y Bienestar Social. Dirección General de Información Estratégica en Salud. Indicadores Básicos de Salud 2016. Asunción: Dirección General de Información Estratégica en Salud; 2017. Disponible en: http:// www.mspbs.gov.py/digies/wp-content/ uploads/2012/01/IBS-Paraguay-2016.pdf

11. Bosch J. Análisis de los motivos de petición de la técnica eutanásica por parte de los enfermos. Cuad Bioética [Internet]. 2003;14(50):61-8. Disponible en: https://www.researchgate.net/publication/28083573_Analisis_de_los_motivos_de_peticion_de_la_tecnica_eutanasica_por_parte_de_los_enfermos

12. Amado E. La despenalización de la eutanasia en Colombia: contexto, bases y críticas. Rev bioética y derecho [Internet]. 2017 [citado 24 de julio de 2017]; Recuperado a partir de: $h t t p: / /$ revistes.ub.edu/ index.php/RBD/article/view/19167

13. López AL. La regulación de la eutanasia, según la ley $\mathrm{N}^{\circ} 20.584$ sobre derechos del paciente. Rev Derecho la PontifUniv Católica Valparaíso XLI. 2013; 505-58.

14. Luley S. «Suicide tourism»: creatingmisleading „scientific" news. J MedEthics [Internet]. 24 de julio de 2015; 41(8):618 LP-619. Recuperado a partir de: http://jme.bmj.com/content/41/8/618.abstract

15. Fischer $\mathrm{S}$, Huber $\mathrm{CA}$, Imhof $\mathrm{L}$, MahrerImhof R, Furter M, Ziegler SJ, et al. Suicide assisted by two Swiss right-todie organizations. J Med Ethics [Internet]. 30 de octubre de 2008; 34(11):810 LP-
814. Recuperado a partir de: http://jme. bmj.com/content/34/11/810.abstract

16. García Hirsch SMI, Guevara Peralta RJ. Percepción de la Eutanasia y factores asociados, en estudiantes de Medicina de la Facultad de Ciencias de la Salud de la Universidad Peruana de Ciencias. 2014 [citado 23 de julio de 2017]; Recuperado a partir de: http://repositorioacademico.upc.edu.pe/upc/handle/10757/322312

17. Ramirez-Rivera J, Cruz J, Jaume-Anselmi F. Euthanasia, assisted suicide and end-oflife care: attitudes of students, residents and attending physicians. $\mathrm{P} R$ Health Sci J 2006 Dec; 25(4):325-329. [citado 19 Septiembre 2017] Disponibleen:http://bases.bireme.br/cgibin/ wxislind.exe/ iah/online/?IsisScript $=$ iah/iah. $x$ is $\&$ src $=$ google \&base $=$ LILACS\&lang $=\quad p \& n e x-$ tAction $=$ lnk\&exprSearch=472096\&indexSearch=ID

18. Casas $M$ de la L, Pichardo LM, Manzano M del C, Torres JC, Gómez Sanchez J. Encuesta piloto comparativa de opinión de médicos, enfermeras y estudiantes de medicina sobre el suicidio asistido en un hospital privado del Distrito Federal. Pers y Bioética [Internet]. 2009;11(2):186-95. Disponible en: http:// personaybioetica.unisabana.edu.co/index. php/personaybioetica/article/view/952

19. Rojas KR. Nivel de conocimientos y actitudes acerda de la eutanasia en internos y médicos residentes de cinco hospitales del departamento de Lambayeque en el año 2015. RegistNacTrabInvestig - Perú [Internet]. 2017 [citado 23 de julio de 2017]; Disponible en: http://www.repositorioacademico.usmp.edu.pe/handle/ usmp/256219.

20. Anneser J, Jox R, Borasio GD. Physician-assisted suicide, euthanasia and palliative sedation: attitudes and knowled- 
ge of medical students. GMS J MedEduc [Internet]. 2016;33(1):1-14. Disponible en: https:/www.ncbi.nlm.nih.gov/pmc/articles/PMC4766939/ 